\title{
Hard Probes Results from the ATLAS Experiment
}

\author{
Alexander Milov*, on behalf of the ATLAS Collaboration ${ }^{\dagger}$ \\ Weizmann Institute of Science, 234 Herzl str., Rehovot 76100, Israel \\ E-mail: alexander.miloveweizmann.ac.il
}

The ATLAS experiment at the LHC has a diverse research program exploring the physics of the heavy ion collisions at the LHC energies. The strength of the ATLAS detector is in the measuring the hard probes that play the crucial role in understanding various physics phenomena helping to unveil the physics nature of the system created in ion collisions. The results of the ATLAS effort over the last year are reviewed in this proceedings that includes the last measurement done with the electroweak probes, a very broad spectrum of measurements performed with jets, the first results on bound and open heavy quark states and results from the ultra-peripheral collisions. The measurements performed by ATLAS span all the collision systems available at the LHC, from small $p p$ and $p+\mathrm{Pb}$ to intermediate and large $\mathrm{Xe}+\mathrm{Xe}$ and $\mathrm{Pb}+\mathrm{Pb}$.

International Conference on Hard and Electromagnetic Probes of High-Energy Nuclear Collisions 30 September - 5 October 2018

Aix-Les-Bains, Savoie, France

* Speaker.

${ }^{\dagger}$ C) 2018 CERN for the benefit of the ATLAS Collaboration. CC-BY-4.0 license 
The ATLAS experiment at the LHC is a universal apparatus that is capable of performing different types of measurements. The strength of the ATLAS detector [1] is in the measurement of jets and electroweak probes that constitute a very important class of probes for the understanding of the Quark Gluon Plasma. The main results from the ATLAS Collaboration worked out in recent time has been presented at the Hard Probes conference and are reviewed in this proceedings.

\section{Electroweak probes}

Electroweak probes in heavy ion collisions can be measured in their initial state, i.e. unaffected by the presence of strongly interacting medium. This makes these probes a powerful tool to assess the initial stage of the collisions, the accuracy of the centrality determination, cold nuclear matter effects, control over the initial energy of jets. These measurements performed using the ATLAS detector are shown in Figure 1 for $Z$ and $W$ bosons and isolated high-energy photons. The $Z$ boson
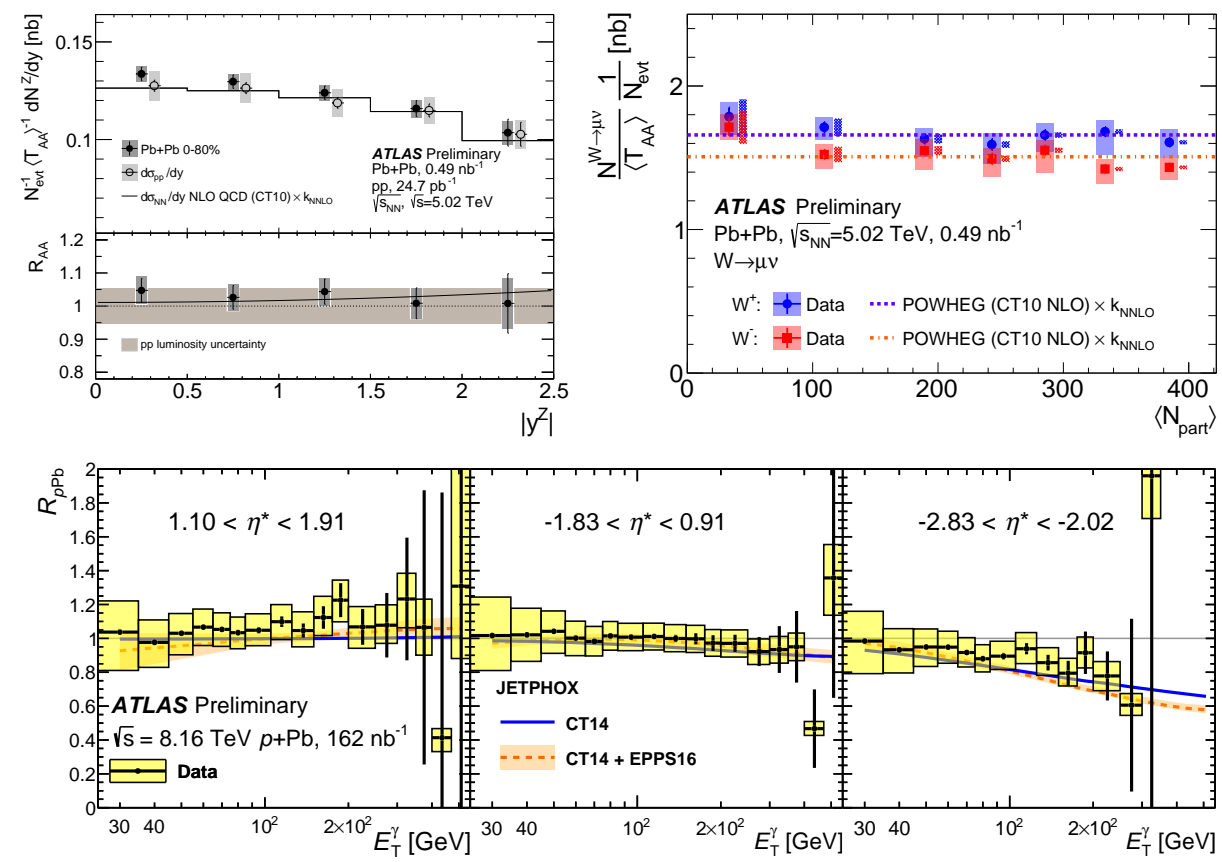

Figure 1: Left: rapidity dependence of the $Z$ boson production [2] in $\mathrm{Pb}+\mathrm{Pb}$. Right: centrality dependence of $W$ boson [3]. Bottom: Momentum dependence of the nuclear modification for photons [4] in $p+\mathrm{Pb}$.

results confirm that the $\mathrm{Pb}+\mathrm{Pb}$ yields divided by the nuclear thickness function are consistent with $p p$ taking into account the isospin effects. Results with the $W$ bosons show that this observation holds for all intervals of measured centrality and results with photons in $p+\mathrm{Pb}$ show that the models generally describe the measurements well. Some deviations are seen for highly energetic photons at high rapidity. Similar deviation from in this region are also seen in $p p$ [4]. As a result, the nuclear modification factor $\left(R_{\mathrm{AA}}\right.$, in this case $\left.R_{p \mathrm{~Pb}}\right)$ shown in Figure 1 is well described by the models. The ATLAS experiment also published a high accuracy measurement of the $Z$ and $W$ production cross sections in $p p$ system at $\sqrt{s}=5.02 \mathrm{GeV}[5]$ as shown in Figure 2 

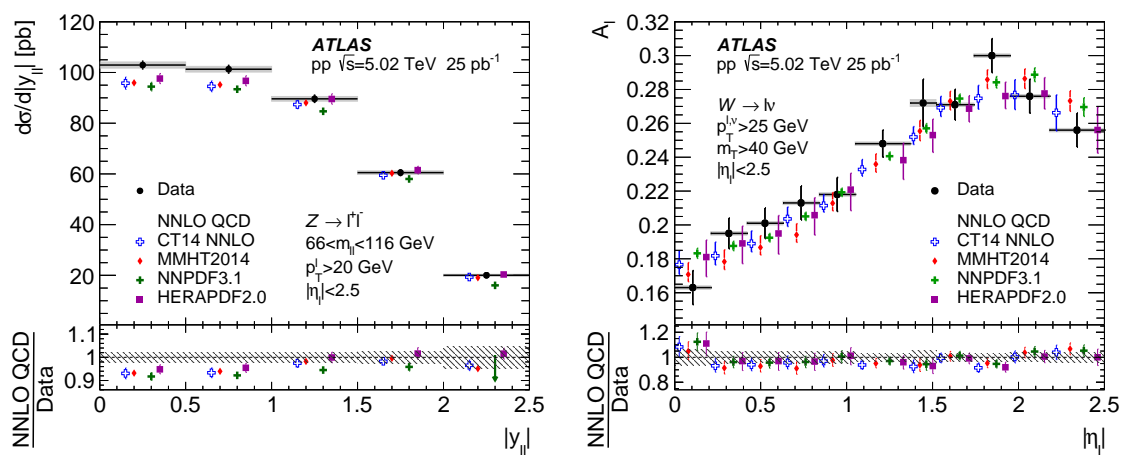

Figure 2: Rapidity distribution of the $Z$ bosons and the asymmetry of leptons from $W$ decays in $p p$ [5].

\section{Quarkonia and open heavy flavor}

The ATLAS experiment measures the suppression of prompt and non-prompt $J / \Psi$ mesons in $\mathrm{Pb}+\mathrm{Pb}$ and $p p$ collisions [6]. The $R_{\mathrm{AA}}$ that is derived from these measurements is shown in Figure 3 as a function of transverse momentum $\left(p_{\mathrm{T}}\right)$ and centrality.
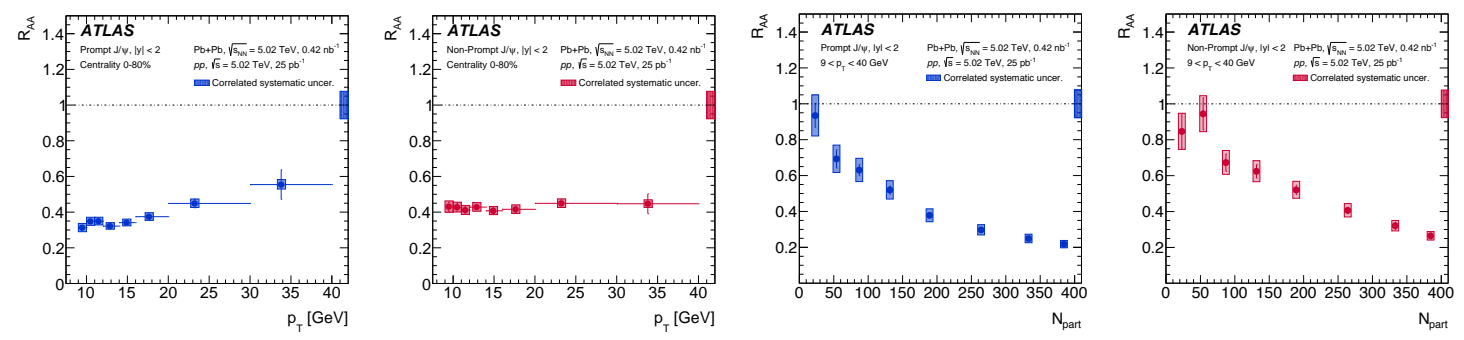

Figure 3: The $R_{\mathrm{AA}}$ for prompt and non-prompt $J / \Psi$ [6]. The left two panels show the $p_{\mathrm{T}}$ dependence and the right two panels show the centrality dependence.

The $p_{\mathrm{T}}$ dependence is different for the prompt and non-prompt components, but overall the charmonia suppression pattern is consistent with that for other hadronic probes. The second harmonic anisotropic coefficient shown in Figure 4 hints at some differences between prompt and
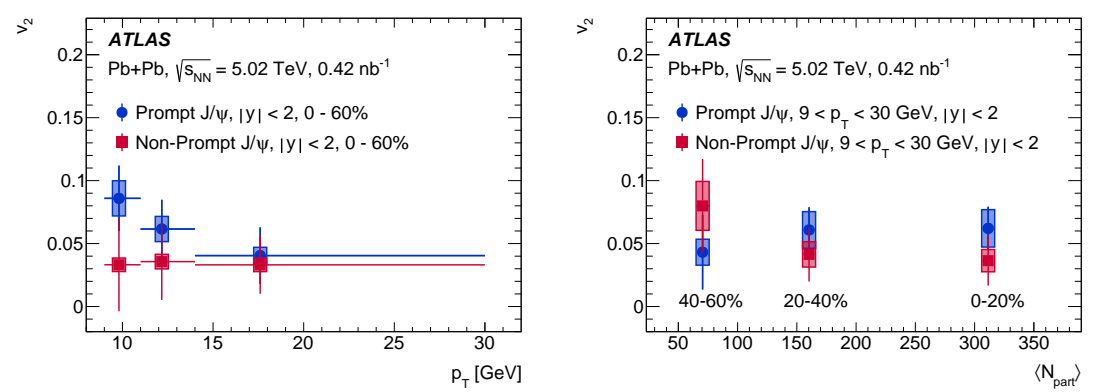

Figure 4: The $2^{\text {nd }}$ anisotropy coefficient for prompt and non-prompt $J / \Psi$ [7]. 
non-prompt components coming from the differences in their production mechanisms. The measured anisotropy is consistent with the regeneration scenario of the $J / \Psi$ production.

Production of $D^{0}$ and $D^{* \pm}$ mesons has been measured by ATLAS in $p+\mathrm{Pb}$ collisions [8] in forward and backward rapidity intervals. No significant asymmetry is observed. The results are presented in Figure 5. The second-order harmonic coefficients for inclusive $D^{* \pm}$ extracted using template fits as shown in the figure.
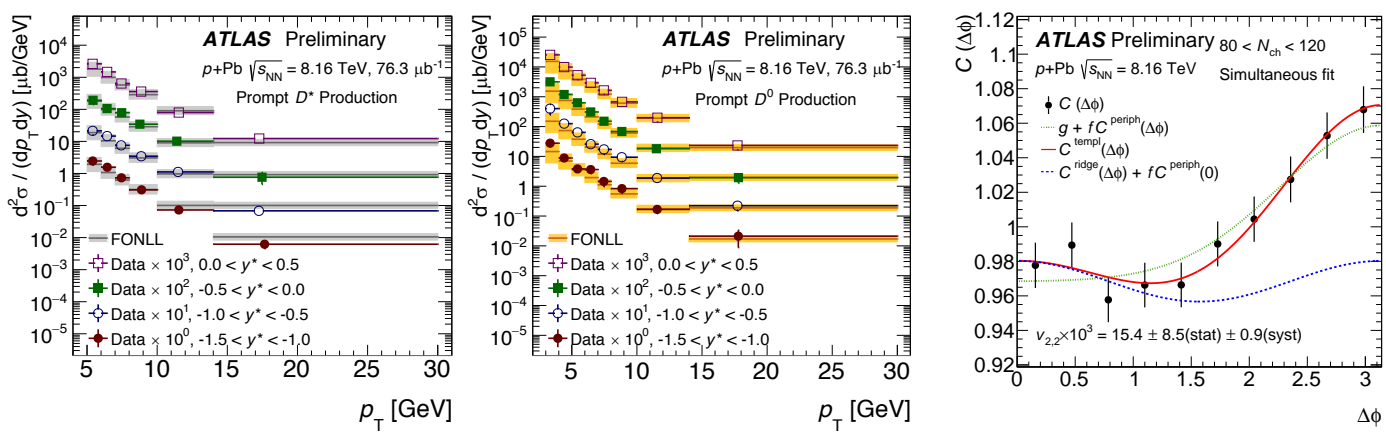

Figure 5: Left and middle: the $D^{*}$ and $D^{0}$ and differential cross section in several rapidity bins. Right: azimuthal anisotropy of $D^{*}$. More results are available in Ref. [8].

\section{Jets and high- $p_{\mathrm{T}}$ hadrons}

The momentum and rapidity dependences of fully corrected jets in all centralities of $\mathrm{Pb}+$ $\mathrm{Pb}$ collisions are available from the ATLAS experiment [9] shown in Figure 6. The results are
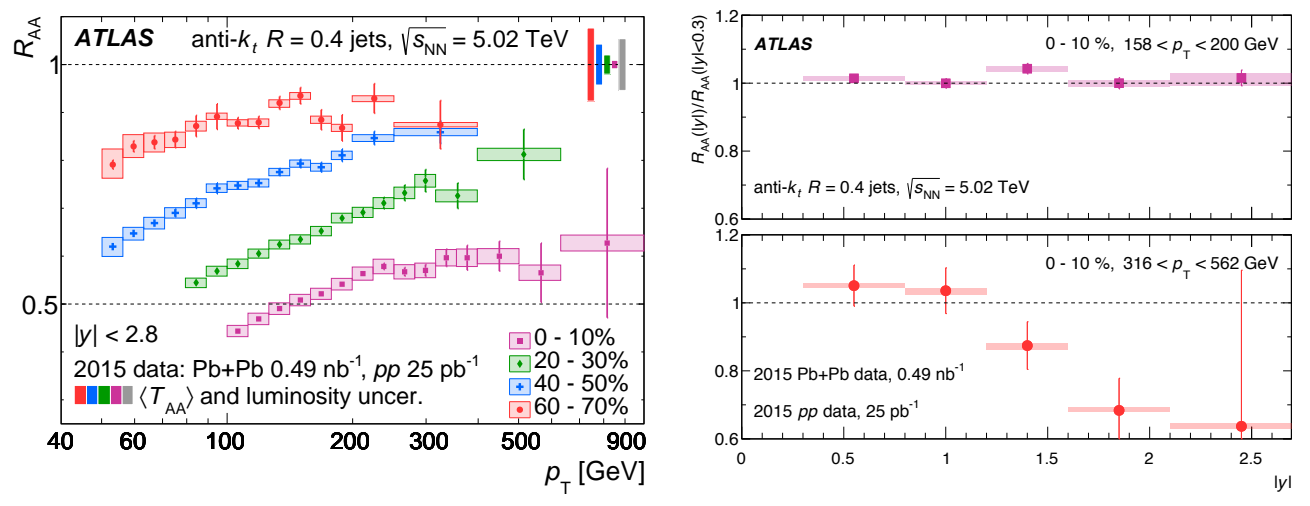

Figure 6: Left: $p_{\mathrm{T}}$ dependence of jet $R_{\mathrm{AA}}$ [9]. Right: same for rapidity.

consistent with the measurements at lower energy [10] but have significantly smaller uncertainties. In all centrality intervals the jet $R_{\mathrm{AA}}$ shows rising $p_{\mathrm{T}}$ dependence at low $p_{\mathrm{T}}$ which then becomes much less pronounced. More energetic jets are shown to be more suppressed at high rapidity compared to lower energy jets that do not show any rapidity dependence. 
Jet fragmentation functions (FF) are measured by ATLAS in $\mathrm{Pb}+\mathrm{Pb}$ and in $p p$ collisions at $\sqrt{s_{\mathrm{NN}}}=5.02 \mathrm{TeV}$ [12]. The ratios of the FF in the two systems is shown in Figure 7. The left panel demonstrates a good agreement with the previous ATLAS measurement performed at the lower energy of $\sqrt{s_{\mathrm{NN}}}=2.76 \mathrm{TeV}$ [11]. The middle and the right panels of the figure show the
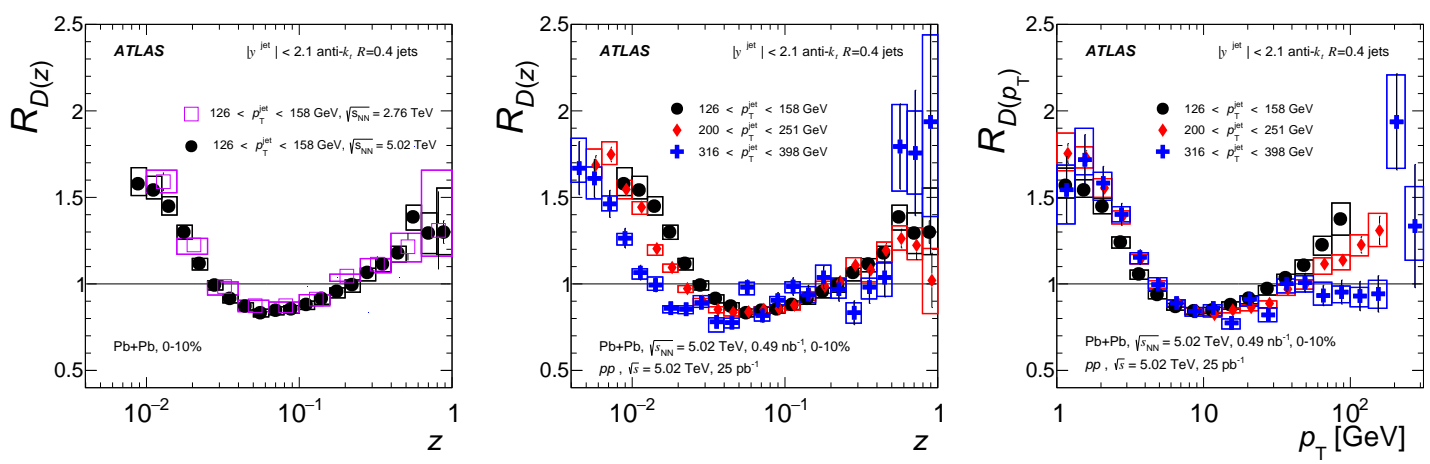

Figure 7: Ratios of the FF measured in $\mathrm{Pb}+\mathrm{Pb}$ and $p p$ collisions. Left: at two different energies [10, 12]. Middle: as a fraction of jet momentum. Right: as a function of particle $p_{\mathrm{T}}$.

modification of the FF vs. the fraction of jet energy carried by the particle and the particle $p_{\mathrm{T}}$ respectively. The plots show that the FF ratios scale with the particle $p_{\mathrm{T}}$, as it would be expected from the medium induced effects.

Figure 8 shows the modification of the $\mathrm{FF}$ in $\mathrm{Pb}+\mathrm{Pb}$ with respect to $p p$ studied as a function of the distance to the jet axis [13]. The measurements reveal that the yield of low- $p_{\mathrm{T}}$ particles increases with increasing momentum of the jet, more low- $p_{\mathrm{T}}$ particles are seen at large radii in central collisions, whereas the yield of high- $p_{\mathrm{T}}$ particles is independent of jet momentum, they get suppressed at small radii.
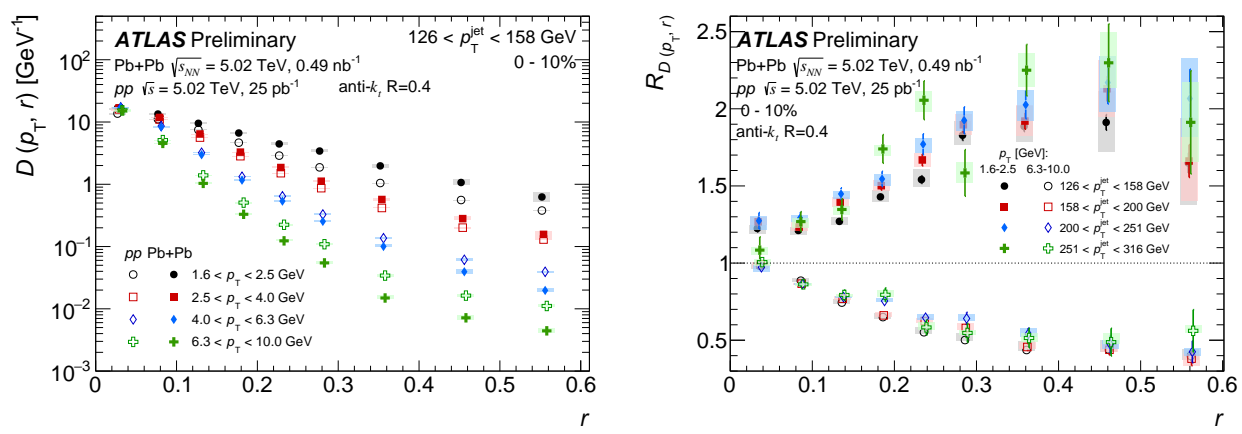

Figure 8: Left: FF vs. the distance to the jet axis in $\mathrm{Pb}+\mathrm{Pb}$ and $p p$ [13]. Right: FF ratios in two systems.

Jet FF is also studied in event where the isolated high- $p_{\mathrm{T}}$ photon is registered [14]. The fraction of quark-jets in such events is higher than in the inclusive sample. The results presented in Figure 9 show that the FF in the two samples are different. A more pronounced flavour dependence is seen in the right panel of the figure corresponding to the most central collisions, whereas in the middle 
panel corresponding to mid-peripheral collisions the FF in the $\gamma$-tagged and inclusive events are similar.
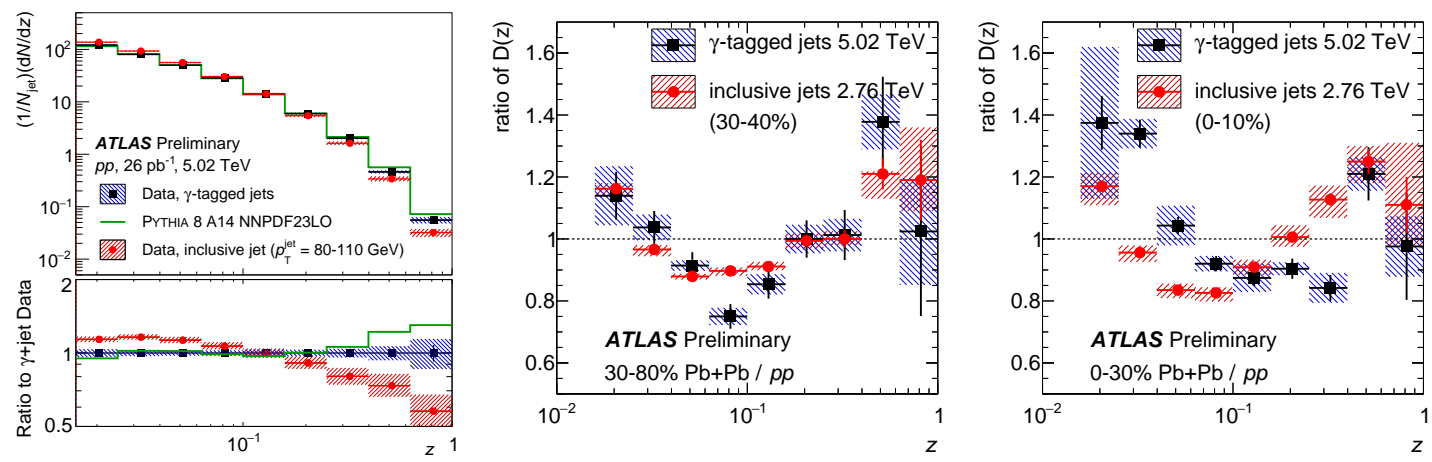

Figure 9: Left: the FF measured in $\gamma$-tagged and inclusive samples and their ratio [14]. Middle and Right are the ratios of the $\mathrm{FF}$ in the $\mathrm{Pb}+\mathrm{Pb}$ and $p p$ in mid-peripheral and central collisions.

One of the greatest advantages of the ATLAS detector in heavy ion studies is its capability to measure boson-jet pairs. This constrains the initial jet energy and allows direct observation of the jet energy loss by measuring back-to-back pairs [15]. The balance of the jet to photon transverse momenta $X_{J \gamma}$ is plotted in Figure 10. The results are compared to the measurements performed in $p p$ collisions at the same energy presented in the left panel. These data produce a distinct peak
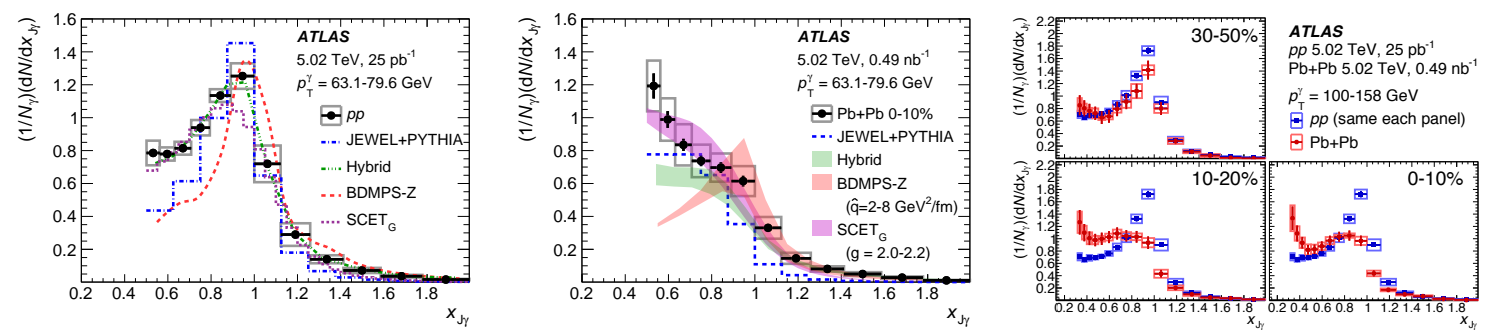

Figure 10: Jet to photon transverse momenta balance $X_{J \gamma}$ [15]. Left and middle panels show $p p$ and central $\mathrm{Pb}+\mathrm{Pb}$ collisions respectively and right panels show the centrality evolution of the momentum balance.

at $X_{J \gamma}$ close to unity, reflecting the momentum conservation. The middle panel is showing the most central $\mathrm{Pb}+\mathrm{Pb}$ collisions where the peak disappears and lower values of $X_{J \gamma}$ rise due to jet in-medium energy loss. Both peak dependencies are compared to the model describing the initial stage effects and the energy loss. The right panel depicts several intermediate centrality classes showing the evolution of the jet energy loss with the size of the system.

In 2017 the LHC provided the first collisions of intermediate mass ions, $\mathrm{Xe}+\mathrm{Xe}$. Although the integrated luminosity of a several hours run constituted only $3 \mu \mathrm{b}^{-1}$ the ATLAS experiment accomplished several important measurements in this new collision system that can be compared to $\mathrm{Pb}+\mathrm{Pb}$ [16]. The momentum balance of the subleading to leading jet $\left(X_{J}\right)$ is shown in Figure 11 for different centrality intervals from the most central on the left to the most peripheral on the right. 

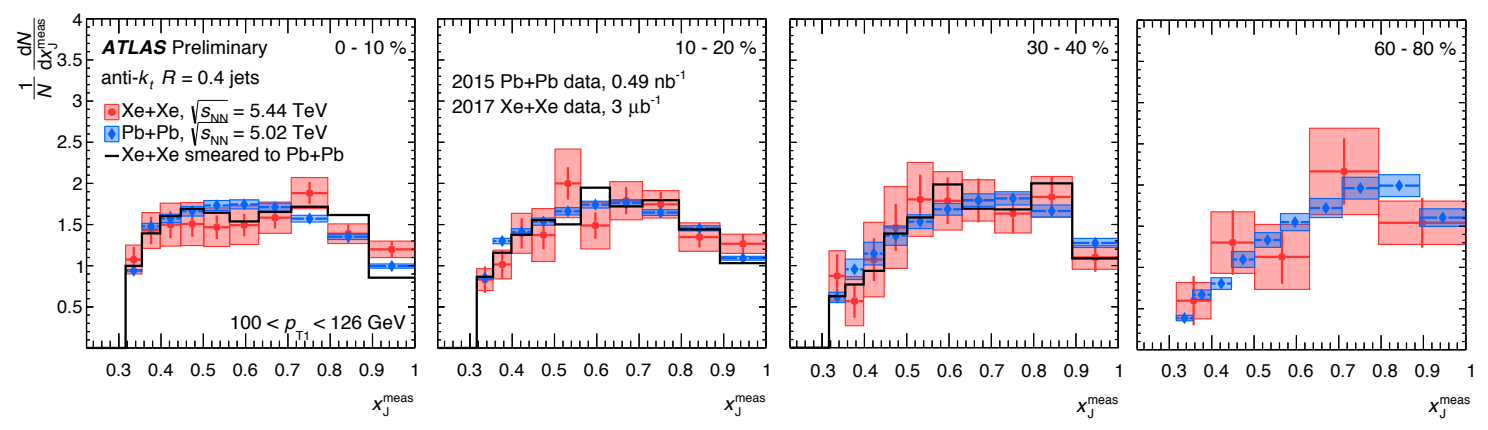

Figure 11: $X_{J}$ distributions measured in $\mathrm{Xe}+\mathrm{Xe}$ and $\mathrm{Pb}+\mathrm{Pb}$ systems for different centrality intervals [16].

The results shown for the $\mathrm{Xe}+\mathrm{Xe}$ system are not unfolded for the detector effects as it is done in $\mathrm{Pb}+\mathrm{Pb}$. Also, the uncertainty of the latter studies is a lot smaller. The comparison shows that the $X_{J}$ distributions in $\mathrm{Xe}+\mathrm{Xe}$ and in $\mathrm{Pb}+\mathrm{Pb}$ are consistent within uncertainties when compared in the same centrality.

The ATLAS experiment also measures the charged hadron spectra out to nearly $100 \mathrm{GeV}$, where the statistics runs out. These spectra allow to construct the $R_{\mathrm{AA}}$ using $p p$ data measured at $\sqrt{s}=5.02 \mathrm{TeV}$ and extrapolated to $\sqrt{s_{\mathrm{NN}}}=5.44 \mathrm{TeV}$, the centre of mass energy for $\mathrm{Xe}+\mathrm{Xe}$ collisions. The $R_{\mathrm{AA}}$ measured in $\mathrm{Xe}+\mathrm{Xe}$ and $\mathrm{Pb}+\mathrm{Pb}$ systems under different conditions [16] shows the best agreement when measured in events with similar activity in the ATLAS Forward Calorimeter. This is shown in the left panel in Figure 12 . The centrality dependence of the $R_{\mathrm{AA}}$
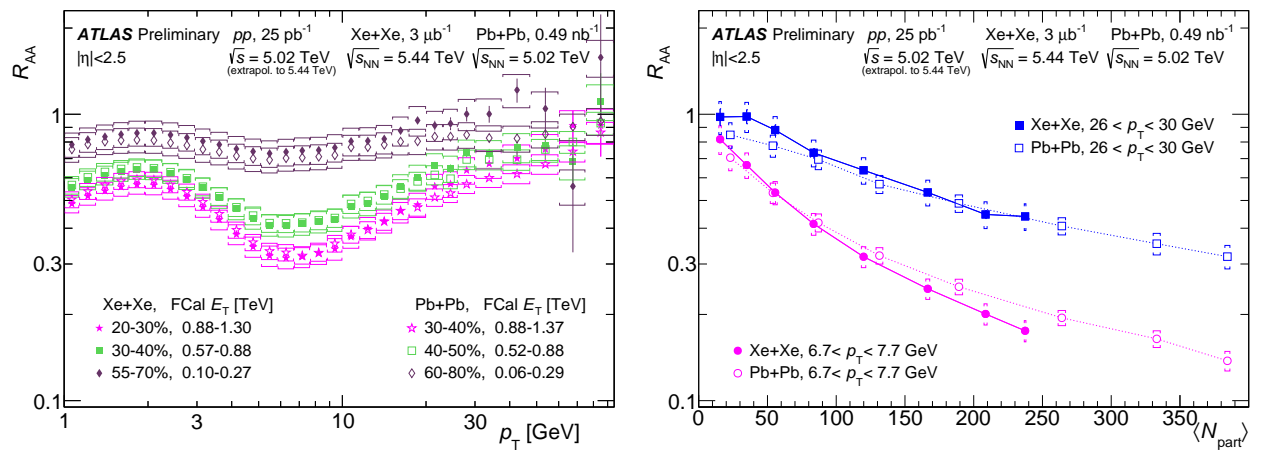

Figure 12: Comparison of the $R_{\mathrm{AA}}$ measured in $\mathrm{Pb}+\mathrm{Pb}$ and in $\mathrm{Xe}+\mathrm{Xe}$ [16]. Left: as a function of $p_{\mathrm{T}}$. Right: as a function of centrality.

shown in the right panel shows a very good agreement between the two systems for hadrons with $p_{\mathrm{T}}$ around $7 \mathrm{GeV}$ and also at higher $p_{\mathrm{T}}$, however, at the lower $p_{\mathrm{T}}$ the agreement is better.

\section{Small systems and ultra-peripheral collisions}

The interest to study the small systems is driven by the observation of the collectivity-like effects in $p+\mathrm{Pb}$ and $p p$ collisions at the LHC which are also seen by RHIC experiments. To make 
another step in these studies one can use the full-energy LHC $p p$ data samples that are coming with the significant pileup background. The ATLAS experiment developed a new technique to subtract the pileup contribution [17]. This is shown in the left panel of Figure 13 presenting the number
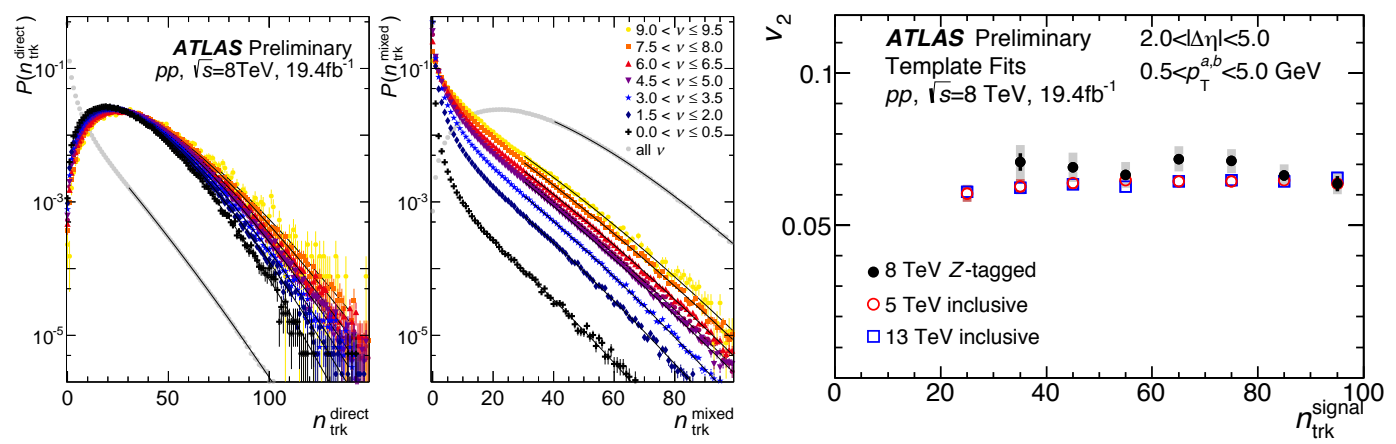

Figure 13: Left: distribution of the number of charged hadrons in the events where $Z$ boson is measured [17]. Middle: Number of pileup tracks in these events. Right: the ratio of two-particle correlations measured in events with the $Z$ boson to inclusive events.

of charged particles in events affected by the pileup. The middle panel shows the distribution of pileup charged particles in those events. Each event selected for this study contained the $Z$ boson and therefore has a significantly different distribution of impact parameters compared to inclusive events. In spite of the difference in the initial geometry of these events and the inclusive events, the magnitudes of two-particle correlations in them are consistent within $8 \pm 6 \%$ at any number of charged tracks in the event.

The ultra-peripheral collisions are typically associated with the electromagnetic interaction at the impact parameters exceeding twice the radius of the colliding ions, i.e. when the hadronic interaction does not occur. The two processes are not however mutually excluding and the electromagnetic interactions may happen simultaneously with the hadronic one. This is measured by the ATLAS experiment [18] at all centralities of the $\mathrm{Pb}+\mathrm{Pb}$ collisions. Different panels of Figure 14 show different centrality intervals. The electromagnetic interactions can be seen as a peak in the

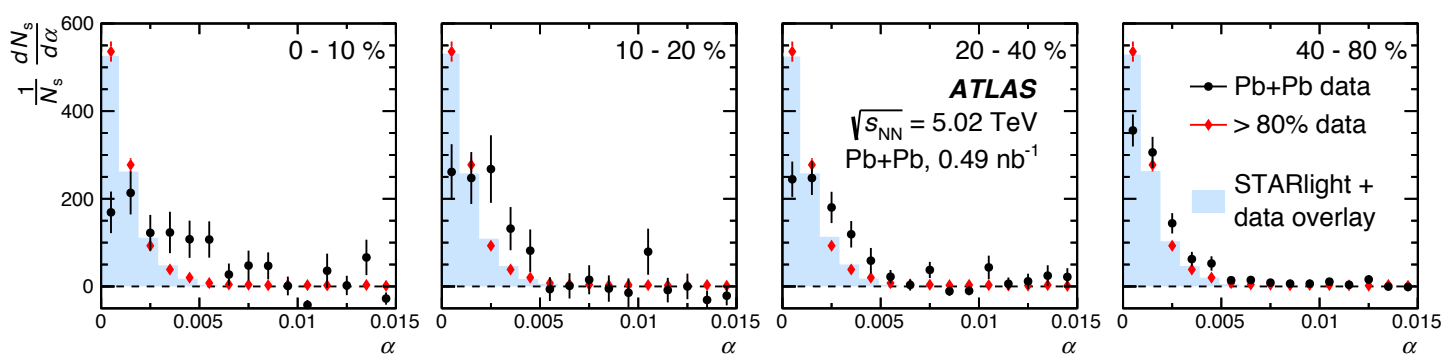

Figure 14: Acoplanarity distribution of two muons with opposite chargers [18] in different centralities.

acoplanarity $\left(\left|\phi^{+}-\phi^{-}\right| / \pi-1\right)$ distribution of two muons with opposite charges. The acoplanarity peak is consistent with the simulation and with the data from ultra-peripheral collisions, however, it 
grows broader in central events, suggesting that the muons scatter from the electric charges present in the medium.

\section{Summary}

The ATLAS experiment presented at the $9^{\text {th }}$ International Conference on Hard and Electromagnetic Probes of High-energy Nuclear Collisions a wealth of new results obtained mainly in the last year. These include the experimental measurements of electroweak probes [2-5], results on the measurement of prompt and non-prompt $J / \Psi$ production properties $[6,7]$ as well as the results on open charm production [8]. Measurements of jets and high- $p_{\mathrm{T}}$ particles in different systems are described in [9-16]. Publications [17] and [18] presents ATLAS results on small systems and ultra-peripheral collisions respectively.

This research of the speaker is supported by the Israel Science Foundation (grant 1065/15), by the Israel Academy of Sciences and Humanities grant number IASAH-ATLAS 712760, and by the MINERVA Stiftung with the funds from the BMBF of the Federal Republic of Germany.

\section{References}

[1] ATLAS Collaboration, JINST 3 (2008) S08003.

[2] ATLAS Collaboration, ATLAS-CONF-2017-010, http://cds.cern.ch/record/2244821

[3] ATLAS Collaboration, ATLAS-CONF-2017-067, http://cds.cern.ch/record/2285571

[4] ATLAS Collaboration, ATLAS-CONF-2017-072, http://cds.cern.ch/record/2285810

[5] ATLAS Collaboration, arXiv: 1810.08424

[6] ATLAS Collaboration, EPJC 9 (2018) 762

[7] ATLAS Collaboration, EPJC 9 (2018) 784

[8] ATLAS Collaboration, ATLAS-CONF-2017-073, http://cds.cern.ch/record/2285811

[9] ATLAS Collaboration, arXiv:1810.05635

[10] ATLAS Collaboration, PRL 114 (2015) 072302

[11] ATLAS Collaboration, EPJC77 (2017) 379

[12] ATLAS Collaboration, PRC98 (2018) 024908

[13] ATLAS Collaboration, ATLAS-CONF-2018-010, http://cds.cern.ch/record/2318869

[14] ATLAS Collaboration, ATLAS-CONF-2017-074, http://cds.cern.ch/record/2285812

[15] ATLAS Collaboration, arXiv: 1809.07280

[16] ATLAS Collaboration, ATLAS-CONF-2018-007, http://cds.cern.ch/record/2318588

[17] ATLAS Collaboration, ATLAS-CONF-2017-068, http://cds.cern.ch/record/2285806

[18] ATLAS Collaboration, PRL 121 (2018) 212301 